\title{
General Current-Mode Analysis Method for Translinear Filters
}

\author{
Jan Mulder, Albert C. van der Woerd, Wouter A. Serdijn, and Arthur H. M. van Roermund
}

\begin{abstract}
Log-domain or translinear filters are regarded as being a promising alternative in the area of low-voltage filter design. To date, most publications have reported on synthesis of translinear filters. Although synthesis is more powerful than analysis, it must go together with a generally applicable analysis method in the same domain. In this paper, a general currentmode analysis method is proposed. By using a current-mode approach, we stay close to the existing theory on static translinear circuits, which might be beneficial in developing a general, possibly more powerful synthesis method.
\end{abstract}

\section{INTRODUCTION}

$\mathbf{T}$ HE APPLICATION OF translinear networks is not restricted to the implementation of static transfer functions. Dynamic transfer functions can be realized as well. The first publication regarding this topic was the introduction of $\log$ domain filters by Adams [1]. The first-order filter described in this publication is in fact a translinear circuit. Some op amps are used, but their main function is to make implementation of linear $V-I$ conversion at the input and $I-V$ conversion at the output feasible, using resistances.

Another example is the general class of filters, called "exponential state-space" filters, published by Frey [2], [3]. Besides the log-domain filters, some other members of this general class are the tanh and sinh filters. As all exponential statespace filters comprise a number of (coupled) translinear loops, they are in fact translinear circuits. For example, the sinh filter reported in [3] comprises nine independent translinear loops. Therefore, the term translinear filter is used throughout this paper.

At present, translinear filters are supposed to be a promising alternative in the area of low-voltage filter design [4]. The trend toward low supply voltages has resulted in a decrease of the dynamic range of filters designed using conventional circuit techniques. This decrease is due to the decrease of the maximum possible voltage swings across the capacitances [5].

A possible solution is companding [6]. The input signal is compressed before the actual filtering operation and is expanded afterwards. As a consequence, the voltages across the capacitances in the filter are nonlinearly related to the input signal, and thus the capacitance voltage swings are much smaller than the corresponding input signal swings. Therefore, the low supply voltage is less restrictive with respect to the maximum allowed input signal.

Manuscript received July 12, 1995; revised February 6, 1996. This paper was recommended by Associate Editor D. A. Johns.

The authors are with the Faculty of Electrical Engineering, Delft University of Technology, 2628 Delft, The Netherlands.

Publisher Item Identifier S 1057-7122(97)02066-7.
Companding is implicitly performed in translinear filters as a consequence of the exponential behavior of the bipolar transistor or the MOS transistor in the subthreshold region. The voltages in a translinear circuit are proportional to the logarithm of the input and output currents.

No definite statements regarding the achievements of translinear filters with respect to dynamic range can be made as yet. The dynamic range is not only determined by the maximum input signal, but also by the noise produced by the circuit. Therefore, the noise behavior of translinear filters has to be examined as well, before any such statements can be made. At present, none of the publications regarding translinear filters has reported on the noise behavior. Besides, measurement results of translinear filters have been published only in [7].

Until now, most publications have reported on synthesis of translinear filters [2]-[4], [7]. Synthesis is more powerful than analysis. However, a useful synthesis method has to be accompanied by a general analysis method in the same domain.

A number of valuable synthesis methods have been reported, differing in the primitive functions on which the filter is mapped. One synthesis path is to use an integrator as the basic function block, and to build the filter by making the right network connections between a number of these integrators [4], [8]. If a log-domain integrator is used, the input and output signal are both currents. The voltage across the capacitance in the integrator is nonlinearly related to the input current, but the transfer function is linear as a whole. Consequently, nonlinearity is only present within the basic integrator block. Filters designed using this approach can be easily analyzed once the integrators are identified.

Another synthesis path uses incomplete translinear loops as its basic building blocks [2], [3], [7]. A transformation is applied to a voltage-mode state-space description of the filter to be realized and the resulting equations are mapped on the basic function blocks. From a translinear point of view, the resulting translinear filter is one inseparable block, i.e., integrators with a linear input-current output-current transfer function cannot be distinguished and consequently, nonlinearity is present at a higher level. The analysis of filters designed according to this synthesis path may be quite complicated, since the filter has to be analyzed in its entirety.

A general analysis method has not been published as yet. The aim of this paper is to propose such a general analysis method, applicable to all translinear dynamic circuits. The method is based on a completely current-mode description of the filter and can be used to obtain the transfer function 
by means of hand calculations. The method might be used as a basis for the development of an alternative, possibly more powerful synthesis theory, in which the signals are directly related to currents.

First, the choice for a current-mode approach to analysis is explained in Section II. Next, in Section III, the general principle behind translinear filters is treated. In Section IV, the analysis method is explained and a second-order low-pass filter, designed by Frey [2], is analyzed in Section V, as an illustration of the method.

\section{CURRENT-Mode APPROACH}

In this paper, a current-mode approach to analysis of translinear filters is used. In our opinion, the term currentmode only applies to the description of the circuit in question. Translinear filters can be described using other methods just as well. An example is the synthesis path published by Frey [2], [3], which starts with a voltage-mode state-space description and uses a transformation to arrive eventually at a translinear implementation of the filter. Therefore, we believe that the circuit is not current mode, but the description of the circuit is current mode.

Though translinear filters can also be described in terms of voltages, there are some reasons why a current-mode approach to analysis is better suited. First, the input signal and the output signal are both currents. Second, the capacitance voltages are nonlinearly related to the currents in the circuit. Using a voltage-mode description, we will have to use logarithmic or exponential functions to describe the circuit. Third, to obtain a voltage-mode differential equation describing the transfer function, an inverse transformation has to be applied [2], [3]. This transformation has to be derived from the circuit, which may be a very complicated task. And last, static translinear circuits are naturally described in terms of products of currents [9]. By using a current-mode approach, the theory developed for static translinear networks will be useful for synthesis of dynamic translinear networks also. The analysis method proposed in this paper can be regarded as a first step in the direction of an alternative, current-mode synthesis method.

\section{PRINCIPLE OF DYNAMIC TRANSLINEAR NETWORKS}

The key to dynamic translinear networks is the exponential behavior of the bipolar transistor or the MOS transistor in the subthreshold region. In this paper, the analysis method will be explained on the basis of the bipolar transistor, since this type of transistor is used in almost all publications on translinear filter circuits. The important difference with respect to translinear filters between the bipolar transistor and the subthreshold MOS transistor is the slope of the exponential characteristic. The slope of the MOST is characterized by the process-dependent parameter $\kappa$ [10], whereas the slope of the bipolar transistor is almost process independent. Therefore, when bipolar transistors are assumed, the equations will be simpler owing to the absence of the subthreshold slope parameter $\kappa$. With some minor modifications, the following theory will also be applicable to subthreshold MOS translinear filters [11].

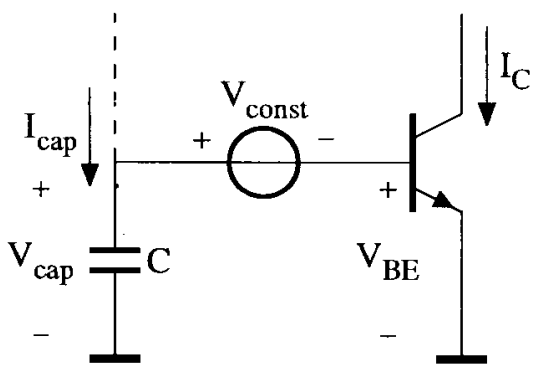

Fig. 1. Principle of dynamic translinear networks.

The collector current $I_{C}$ of a bipolar transistor is in good approximation given by

$$
I_{C}=I_{s} \mathrm{e}^{V_{B E} / U_{T}}
$$

where $I_{s}$ is the saturation current, $V_{B E}$ is the base-emitter voltage and $U_{T}$ is the thermal voltage $k T / q$. The derivative of an exponential function is equal to the exponential function itself multiplied by the derivative of its argument. If we differentiate the collector current, (1), with respect to time, the result is

$$
\dot{I}_{C}=\frac{\dot{V}_{B E}}{U_{T}} I_{C}
$$

where the dot represents differentiation with respect to time.

The time derivative of $V_{B E}$ is very similar to the equation describing the current $I_{c a p}$ through a capacitance $C$, which is given by

$$
I_{\text {cap }}=C \dot{V}_{\text {cap }}
$$

where $V_{c a p}$ is the voltage across the capacitance. If we multiply $\dot{V}_{B E}$ in (2) by $C$, the product $C \dot{V}_{B E}$ can be regarded as the current through a capacitance $C$ with a voltage $V_{B E}$ across it. This is illustrated in Fig. 1. Equation (2) can thus be written as [9], [4]:

$$
\begin{aligned}
C U_{T} \dot{I}_{C} & =I_{C} \cdot C \dot{V}_{B E} \\
& =I_{C} \cdot I_{\text {cap }}
\end{aligned}
$$

The dimension of both sides of (4) is $[A]^{2}$. The right-hand side (RHS) of (4) consists of a multiplication of two currents. A collector current is multiplied by a capacitance current. The derivative of the voltage $V_{\text {cap }}$ across the capacitance is equal to the derivative of the base-emitter voltage $V_{B E}$ of the transistor: $\dot{V}_{\text {cap }}=\dot{V}_{B E}$. Thus, $V_{\text {cap }}$ is equal to $V_{B E}+V_{\text {const }}$, where $V_{\text {const }}$ is an arbitrary constant voltage. This explains the voltage source $V_{\text {const }}$ in Fig. 1 .

Translinear loops are described by products of collector currents of the transistor currents comprising the translinear loop [12]. The loop equation is given by

$$
\prod_{c w} I_{C}=\lambda \prod_{c c w} I_{C}
$$

where $\lambda$ is the emitter area ratio and the products on the left-hand side (LHS) and RHS are over the clockwise and counterclockwise connected transistors, respectively.

From this description, it is clear that the RHS of (4) can easily be realized in translinear technology, as part of a 
translinear loop. Then, the term $I_{C} \cdot I_{c a p}$ can be interpreted as a translinear realization of the LHS of (4), $C U_{T} \dot{I}_{C}$, the derivative of the current $I_{C}$.

Clearly, the factor $C U_{T}$ is on the LHS. This implies that it is not a part of the translinear realization and thus it has to be a part of the transfer function to be realized. As a consequence, the transfer function will be temperature dependent through $U_{T}$. This temperature dependency can be compensated for by making some (or all) currents PTAT [4].

\section{EXPRESSIONS FOR CAPACITANCE CURRENTS}

In developing a general analysis method for dynamic translinear networks, the first thing to be done is to get a clear picture of the difference between static and dynamic translinear circuits. In other words: Why can't we use the analysis methods developed for static translinear networks, e.g., [9]? To answer this question we will have to find the fundamental difference between static and dynamic translinear circuits.

We will now examine the general analysis method for static translinear networks. The first step is to write down the system of all independent equations describing the circuit in question. To a first approximation, base currents are neglected, since these are a second-order effect. The equations fall into two groups: the Kirchhoff's Voltage Law (KVL's) and the Kirchhoff's Current Law (KCL's) .

The KVL's are the loop equations, the number of independent KVL's is equal to the number of independent translinear loops. As a consequence of the translinear principle, the KVL's can be described by products of collector currents [12].

The KCL's are set up for each of the nodes of the circuit. The KCL's are linear equations in the collector currents, input currents, bias currents and output currents. In general, in hand calculations, the KCL's are directly substituted in the currentmode KVL's.

The second step in the analysis of a static translinear circuit is to solve the system of loop equations to obtain the transfer function of the circuit.

Now, the difference between static and dynamic translinear circuits comes forward in writing down the KCL's. In dynamic networks, the KCL's are linear equations not only in the collector currents, input currents, bias currents and output currents, but also in the capacitance currents. A capacitance current can be viewed as a special kind of bias current, which is derived from the input and/or output currents. It can be single-ended or floating.

During analysis, the capacitance currents are unknown, and, therefore, the set of loop equations cannot be solved unless additional expressions can be found for these capacitance currents. This is exactly the problem to be solved in order to obtain a general analysis method for dynamic translinear networks. In order to eliminate an unknown variable from a system of equations it is, in general, necessary to have at least two independent expressions in the unknown variable. A first expression for the capacitance currents is found from the KCL's.

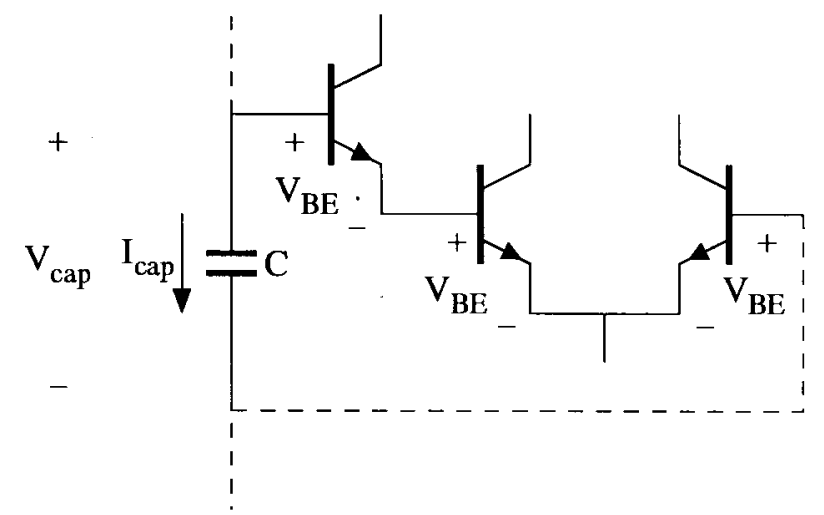

Fig. 2. A capacitance in (a part of) a translinear loop.

We now describe a general method to derive an independent second expression. In a translinear filter, a capacitance is always connected between two nodes of the translinear loops. As a consequence, the capacitance is connected in series with a certain number of base-emitter junctions. This is illustrated in Fig. 2. Though any translinear loop comprises an even number of junctions, the number of junctions in series with the capacitance may be odd or even. Also, the junctions in this loop may be connected in the same direction, in opposite directions or in a combination of both.

As the capacitance and the junctions together comprise a loop, the capacitance voltage can be expressed in terms of the junction voltages, using the KVL:

$$
\begin{aligned}
V_{\text {cap }} & =\sum_{i} \pm V_{B E, i} \\
& =U_{T} \sum_{i} \pm \ln \left(\frac{I_{C, i}}{\lambda_{i} I_{s}}\right)
\end{aligned}
$$

where $I_{C, i}$ are the collector currents corresponding to the baseemitter voltages $V_{B E, i}$ and $\lambda_{i}$ are the relative emitter area ratios. The sign of each voltage $V_{B E, i}$ in this equation is dependent on the orientation of the base-emitter junction in the loop.

The current through the capacitance is equal to the derivative $\dot{V}_{\text {cap }}$ multiplied by the value $C$ of the capacitance. Differentiating (6) and multiplying it by $C$ thus yields the capacitance current $I_{\text {cap }}$ :

$$
I_{\text {cap }}=C U_{T} \sum_{i} \pm \frac{\dot{I}_{C, i}}{I_{C, i}}
$$

This equation is the basis of the general analysis method for dynamic translinear circuits!

The equation gives an expression for each of the capacitance currents which is independent from the loop equations and KCL's comprising the rest of the system of equations. With the aid of these additional equations, the system can be solved, i.e., the transfer function can be obtained.

Like the loop equations and the KCL's, the expressions resulting from (7) are current mode. Thus, a dynamic translinear circuit can be described by a system of equations completely in terms of currents. 


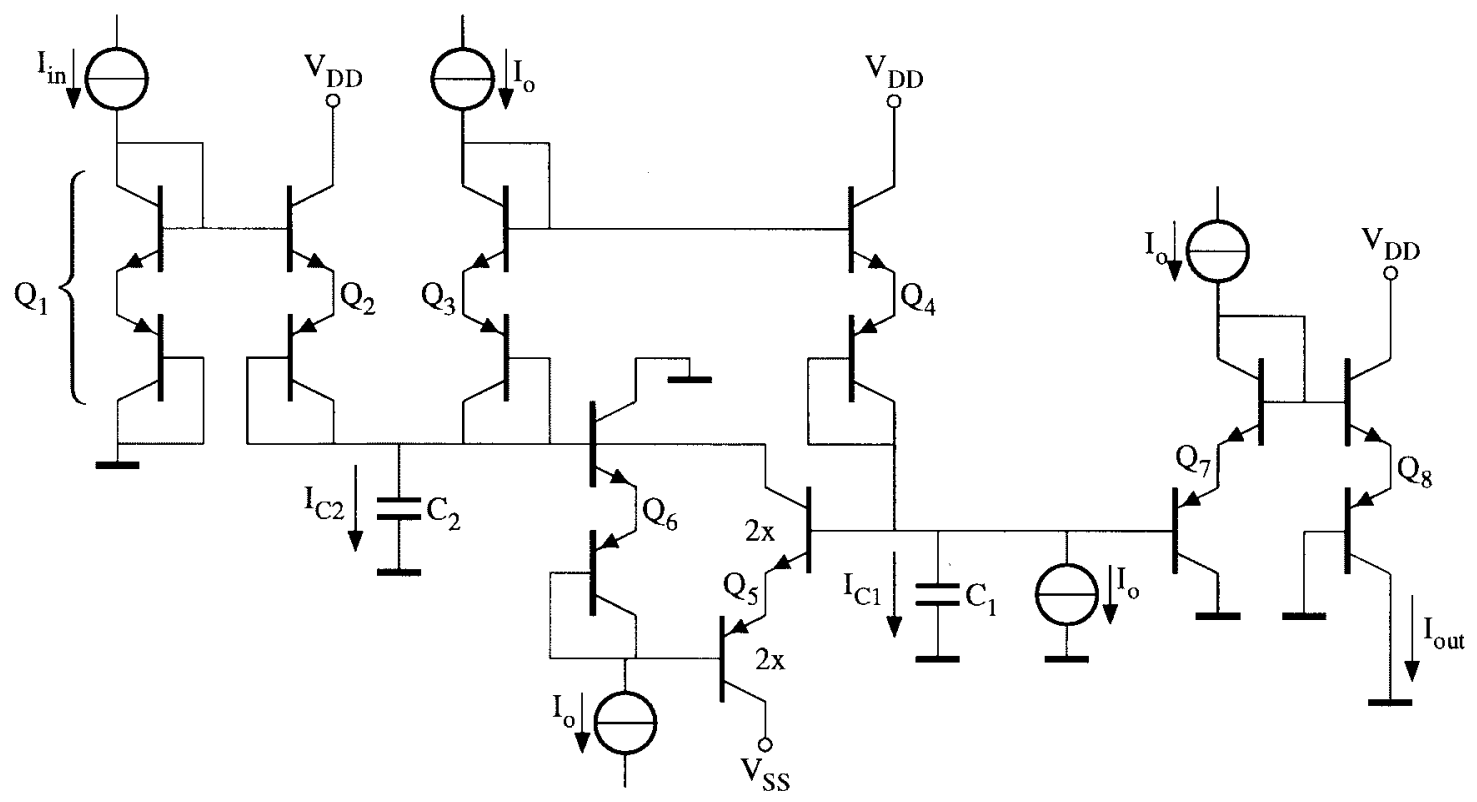

Fig. 3. Second-order low-pass filter by Frey.

Each capacitance comprises at least two loops with the junctions in a translinear circuit. Therefore, more than one expression for a capacitance current can always be derived. With respect to the other equations necessary to describe the circuit, these capacitance current expressions are dependent. Consequently, (7) has to be applied to each capacitance just once. The other possible capacitance current expressions are superfluous.

Direct current collector currents flowing through some of the transistors in a capacitance-junction(s) loop are automatically eliminated from (7), since the derivative of a constant equals zero.

\section{AN ANALYSIS EXAMPLE}

As an illustration of the proposed analysis method, we analyze the second-order low-pass filter designed by Frey [2]. The filter is shown in Fig. 3. All transistors comprising the translinear loops are compound transistors, to simplify biasing of the circuit, but for analysis purposes they can simply be treated as single transistors, changing $U_{T}$ in (1) into $2 U_{T}$. The filter shown in Fig. 3 consists of two coupled translinear loops. The first loop comprises transistors $Q_{1}-Q_{2}-Q_{3}-Q_{4}-Q_{7}-$ $Q_{8}$, the second loop comprises $Q_{3}-Q_{4}-Q_{5}-Q_{6}$. For both loops, a loop equation can be derived. Direct substitution of the KCL's to express the loop equations in terms of $I_{\mathrm{in}}, I_{\mathrm{out}}, I_{o}$, and the capacitance currents $I_{C_{1}}$ and $I_{C_{2}}$, as much as possible, yields:

$$
\begin{aligned}
I_{\mathrm{in}} I_{o} I_{o} & =I_{2}\left(I_{C_{1}}+I_{o}\right) I_{\mathrm{out}} \\
2 I_{o} I_{o} & =\left(I_{C_{1}}+I_{o}\right)\left(I_{2}+I_{o}-I_{C_{2}}\right)
\end{aligned}
$$

where the factor 2 is a consequence of the emitter area scaling of $Q_{5}$, and $I_{2}$ is the collector current of $Q_{2}$. This is the only transistor current which cannot be expressed as a linear combination of $I_{\mathrm{in}}, I_{\mathrm{out}}, I_{o}, I_{C_{1}}$ and/or $I_{C_{2}}$.
Next, two expressions for $I_{C_{1}}$ and $I_{C_{2}}$ are derived. In order to apply (7), two loops have to be chosen. For $I_{C_{1}}$ the loop $C_{1}-Q_{7}-Q_{8}$ is used, and for $I_{C_{2}}$ the loop $C_{2}-Q_{3}-Q_{4}-Q_{7}-Q_{8}$ is used, yielding

$$
\begin{aligned}
& I_{C_{1}}=C_{1}\left(2 U_{T}\right) \frac{\dot{I}_{\text {out }}}{I_{\text {out }}} \\
& I_{C_{2}}=C_{2}\left(2 U_{T}\right)\left(\frac{\dot{I}_{C_{1}}}{I_{C_{1}}+I_{O}}+\frac{\dot{I}_{\text {out }}}{I_{\text {out }}}\right) .
\end{aligned}
$$

The collector currents of $Q_{3}$ and $Q_{7}$ are equal to the constant bias current $I_{o}$. Since the derivative of a constant is zero, the collector currents of $Q_{3}$ and $Q_{7}$ do not appear in the equations for $I_{C_{1}}$ and $I_{C_{2}} . Q_{3}$ and $Q_{7}$ can in this context be regarded as dc voltage sources, as in Fig. 1.

The particular choice for the two capacitance-junctions loops, used to derive (10) and (11), has led to relatively simple expressions for $I_{C_{1}}$ and $I_{C_{2}}$. Other choices result in the appearance of the derivative $\dot{I}_{C_{1}}$ on the RHS of (10) and/or $\dot{I}_{C_{2}}$ on the RHS of (11). In these cases, expressions for $\dot{I}_{C_{1}}$ and/or $\dot{I}_{C_{2}}$ have to be calculated from (8) and (9) and substituted in the RHS of (10) and (11).

Now, the system of (8)-(11) has to be solved to obtain the transfer function of the filter. First, (10) and its derivative can be used to eliminate $I_{C_{1}}$ and $\dot{I}_{C_{1}}$ from (8), (9), and (11). $I_{C_{2}}$ thus becomes:

$$
I_{C_{2}}=C_{2}\left(2 U_{T}\right) \frac{C_{1}\left(2 U_{T}\right) \ddot{I}_{\mathrm{out}}+I_{o} \dot{I}_{\mathrm{out}}}{C_{1}\left(2 U_{T}\right) \dot{I}_{\mathrm{out}}+I_{o} I_{\mathrm{out}}} .
$$

Substitution of this equation for $I_{C_{2}}$ in the loop equations (8) and (9) yields two expressions containing two unknowns: $I_{\text {out }}$ and $I_{2}$. Since these two expressions are linear in $I_{2}$, this current can easily be eliminated. The result is a linear differential equation, expressing the transfer function from the 
input current to the output current:

$$
\begin{aligned}
& C_{1} C_{2}\left(2 U_{T}\right)^{2} \ddot{I}_{\text {out }}+\left(C_{2}-C_{1}\right)\left(2 U_{T}\right) I_{0} \dot{I}_{\text {out }} \\
& \quad+I_{0}^{2} I_{\text {out }}=I_{0}^{2} I_{\text {in. }} .
\end{aligned}
$$

Clearly, the terms $C_{1} U_{T}$ and $C_{2} U_{T}$ are part of the transfer function, as explained in Section III.

This example demonstrates the applicability of the general analysis method for dynamic translinear circuits proposed in this paper.

\section{CONCLUSIONS}

The synthesis methods for translinear filters published to date are not attended by a generally applicable analysis method. In this paper, a general analysis method was proposed. Translinear filters can be analyzed completely in terms of currents. By choosing a current-mode approach, we stay close to the existing theory regarding static translinear circuits. Consequently, it might be possible to exploit this theory in developing an alternative synthesis method for dynamic translinear circuits, in which the relevant signals are related to currents. Research is going on in this direction and the results will be published in subsequent papers.

The difference between static and dynamic translinear circuits is in the capacitance currents, which can be regarded as a special kind of bias currents. To find the transfer function of a translinear filter, expressions for the capacitance currents, which are independent from the loop equations and KCL's, have to be derived from the circuit. A general method to obtain these capacitance currents equations was derived. The method will be useful for hand calculations. The proposed method was illustrated by performing a current-mode analysis of a translinear second-order low-pass filter, designed by Frey.

\section{REFERENCES}

[1] R. W. Adams, " Filtering in the log domain," 63rd Convention A.E.S. LA, preprint 1470, May 1979.

[2] D. R. Frey. "Log-domain filtering: An approach to current-mode filtering," Inst. Elec. Eng. Proc., Pt. G, vol. 140, no. 6, pp. 406-416, Dec. 1993.

[3] , "A general class of current mode filters," in Proc. ISCAS, vol. 2, 1993, pp. 1435-1438.

[4] E. Seevinck, "Companding current-mode integrator: A new circui principle for continuous-time monolithic filters," Electron. Lett., vol. 26, no. 24, pp. 2046-2047, Nov. 1990.

[5] G. Groenewold, " Optimal dynamic range integrated continuous-time filters," Ph.D. dissertation, Delft Univ. Technol., 1992.

[6] Y. P. Tsividis, V. Gopinathan, and L. Toth, " Companding in signal processing," Electron. Lett., vol. 26, no. 17, pp. 1331-1332, Aug. 1990.

[7] D. Perry and G. W. Roberts, " Log-domain filters based on LC ladder synthesis," in Proc. ISCAS, vol. 1, 1995, pp. 311-314.

[8] M. Punzenberger and C. Enz, " Low-voltage companding current-mode integrators," in Proc. ISCAS, vol. 3, 1995, pp. 2112-2115.

[9] E. Seevinck, Analysis and Synthesis of Translinear Integrated Circuits. Amsterdam: Elsevier, 1988.
[10] A. Pavasovic, " Subthreshold region MOSFET mismatch analysis and modeling for analog VLSI systems," Ph.D. dissertation, John Hopkins Univ., Baltimore, MD, 1991.

[11] C. Toumazou and T. S. Lande, "Micropower log-domain filter for electronic cochlea," Electron. Lett., vol. 30, no. 22, pp. 1839-1841, Oct. 1994.

[12] B. Gilbert, "Translinear circuits: A proposed classification," Electron. Lett., vol. 11, no. 1, pp. 14-16, Jan. 1975.

Jan Mulder was born on July 7, 1971, in Medemblik, The Netherlands. He received the M.Sc. degree in electrical engineering from the Delft University of Technology in 1994. Since 1994 he has been working toward the Ph.D. degree on static and dynamic translinear analog integrated circuits, at the Electronics Research Laboratory.

Albert C. van der Woerd was born in 1937 in Leiden, The Netherlands. In 1977 he received the "ingenieurs" (M.Sc.) degree in electrical engineering from the Delft University of Technology, Delft, The Netherlands. He received the Ph.D. degree in 1985.

From 1959 to 1966 he was engaged in research on and the development of radar and TV circuits at several industrial laboratories. In 1966 he joined the Electronics Research Laboratory of the Faculty of Electrical Engineering of the Delft University of Technology. For the first 11 years he carried out research on electronic musical instruments. For the next eight years his main research interest was carrier domain devices. More recently he has specialized in the field of low-voltage low-power analog circuits and systems. He currently teaches design methodology.

Wouter A. Serdijn was born in 1966 in Zoetermeer, The Netherlands, He received the "ingenieurs" (M.Sc.) degree from the Delft University of Technology in 1989, and the Ph.D. degree in 1994 from the same university.

His research interests include low-voltage, ultra-low-power, and dynamictranslinear analog integrated circuits along with circuits for hearing instruments and pacemakers. He is coeditor and coauthor of the book Analog IC Techniques for Low-Voltage Low-Power Electronics (Delft: Delft University Press, 1995), and of the book Low-Voltage Low-Power Analog Integrated Circuits (Boston, MA: Kluwer Academic, 1995). He authored and coauthored more than 40 publications. He teaches analog electronics for industrial designers, analog IC techniques, and electronic design techniques.

Arthur H. M. van Roermund was born in Delft, The Netherlands, in 1951. He received the M.Sc. degree in electrical engineering in 1975 from the Delft University of Technology and the Ph.D. degree in applied sciences from the K.U. Leuven, Belgium, in 1987.

From 1975 to 1992 he was with the Philips Research Laboratories in Eindhoven. He has worked in the Consumer Electronics Group on design and integration of analog circuits and systems, especially switched-capacitor circuits, and in 1987, he joined the Visual Communications Group where he has been engaged in video architectures and digital video signal processing. From 1987 to 1990 he was project leader of the Video Signal Processor project and from 1990 to 1992, he was the leader of a Multi-Window Television project. Since 1992 he has been a full professor with the Electrical Engineering Department, Delft University of Technology where he is heading the Electronics Research Laboratory. He is also group leader of the Electronics Group and coordinator of the Circuits and Systems Section of DIMES: the Delft Institute of Micro Electronics and Submicron technology, which is a cooperation between research groups on microelectronics, technology and technology-related physics. 